\title{
NOTE
}

\section{Friction Dynamics on Human Skin Surfaces}

\section{Yoshimune Nonomura* and Haruka Ouchi}

Department of Biochemical Engineering, Graduate School of Science and Engineering, Yamagata University, 4-3-16 Jonan, Yonezawa 992-8510, JAPAN

\begin{abstract}
This study includes an evaluation of friction on the fingers, palms, and forearms of 20 subjects using a sinusoidal motion friction evaluation system. The effects of the changing water content in the stratum corneum at each position as well as the skin elastic modulus on the friction parameters were analyzed to show the factors governing friction dynamics. We observed a significant delay time $(\delta)$ and a stick-slip phenomenon during the sinusoidal motion friction processes. These dynamic phenomena are due to the softness and viscoelastic properties of human skin. The findings regarding the response of human skin to frictional stimulation under accelerated conditions facilitates a better understanding of the dynamic and mechanical properties of human skin.
\end{abstract}

Key words: friction, human skin, sinusoidal motion, cosmetics

\section{Introduction}

The skin is a layered, soft material comprising the epidermis, dermis, and subcutaneous tissue. Its surface is covered with the stratum corneum, which comprises keratin protein, intercellular lipid, and water ${ }^{1)}$. The friction properties of human skin depend on the differences in the moisture content and mechanical properties of each layer. Additionally, the surface exhibits a hierarchical rough structure comprising crista cutis, crevices, pores, and wrinkles $^{2-4)}$. These characteristics can be demonstrated by a power law found between the frictional and normal forces ${ }^{5)}$. According to the law, the friction coefficient of moist or wet skin is much larger than that of dry skin ${ }^{6)}$. Some thickeners, surfactants, and silicone oils change the friction profile on the skin and hair surfaces and further lead to a slimy and smooth feeling ${ }^{7-9)}$.

Conventional friction evaluations are performed at a constant speed. However, the mechanical behavior of soft materials such as skin and hair depends on both the compressive force and shear rate ${ }^{10)}$. We have developed a sinusoidal motion friction evaluation system that evaluates the dynamics of any friction phenomenon occurring on soft material surfaces. Further, we propose a two-step nonlinear model comprising a pre-sliding process and a dynamic friction process ${ }^{11-14}$. Additionally, we have evaluated the friction of two gel substrates and showed that the friction pattern systematically changes with the hardness of the

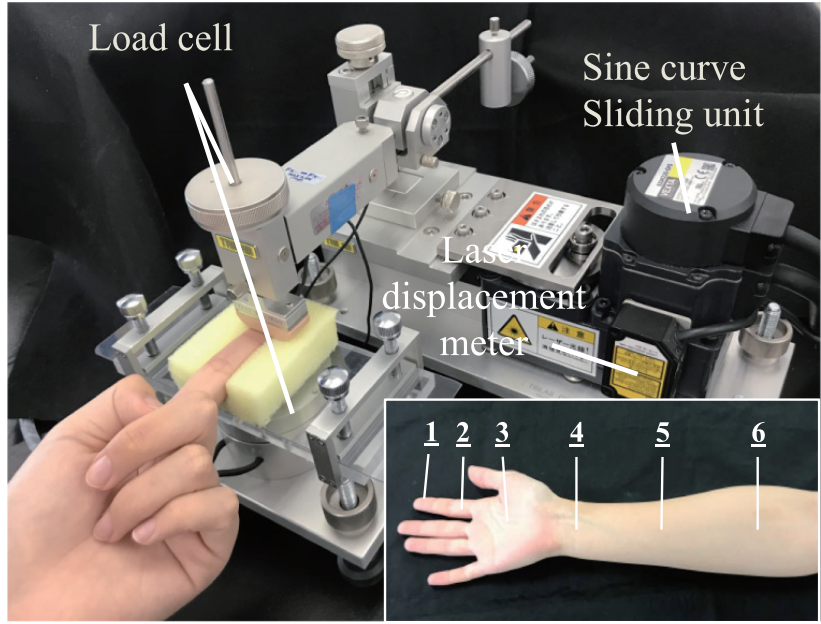

Fig. 1 Sinusoidal-movement friction evaluation system and the evaluation points $\underline{1}-\underline{6}$ on skin surface (inserted image).

gel $^{15,16)}$.

This study includes an evaluation of friction on the fingers, palms, and forearms of 20 subjects using a sinusoidal motion friction evaluation system(Fig. 1). The skin surfaces at points $\underline{1} \underline{-} \underline{6}$ were rubbed with a urethane contact probe. The effects of the changing water content in the stratum corneum at each position as well as the skin elastic

\footnotetext{
* Correspondence to: Yoshimune Nonomura, Department of Biochemical Engineering, Graduate School of Science and Engineering, Yamagata University, 4-3-16 Jonan, Yonezawa 992-8510, JAPAN E-mail: nonoy@yz.yamagata-u.ac.jp Accepted February 14, 2020 (received for review January 15, 2020) Journal of Oleo Science ISSN 1345-8957 print / ISSN 1347-3352 online http://www.jstage.jst.go.jp/browse/jos/ http://mc.manusriptcentral.com/jjocs
} 


\section{Y. Nonomura and H. Ouchi}

modulus on the friction parameters were analyzed to show the factors governing friction dynamics. The findings regarding the response of human skin to frictional stimulation under accelerated conditions facilitates a better understanding of the dynamic and mechanical properties of human skin. Additionally, they elucidate the mechanisms of the tactile stimulation and design of clothes, cosmetics, and pharmaceuticals.

\section{Experimental}

\subsection{Friction evaluation}

All evaluations were conducted according to the principles expressed in the Declaration of Helsinki. The responsible party at Yamagata University confirmed that the ethics and safety of the present test were acceptable. Informed consent was obtained from all subjects. The skin friction of the upper limbs of the subjects comprising 10 men and women aged 20-24 years was evaluated. The measurement sites were the fingertip of the index finger (1), base of the finger (2), palm (ㅇ) , and three points on the forearm (는 $\underline{6}$ ) (Fig. 1). Figure 1 shows an image of a sinusoidal motion friction evaluation system ${ }^{11,12)}$. The friction force, when human skin was rubbed with a contact probe, was measured using this system. Fingerprint-like grooves with a depth of $0.15 \mathrm{~mm}$ are carved on the surface of this contact probe at intervals of $0.5 \mathrm{~mm}$. The measurement conditions are as follows: Sliding width $= \pm 5 \mathrm{~mm}$, vertical force $=0.98 \mathrm{~N}$, number of reciprocations $=11$ times, disk rotation speed $=2.1 \mathrm{rad} \mathrm{s}^{-1}$, maximum velocity $=10 \mathrm{~mm} \mathrm{~s}^{-1}$, temperature $=25 \pm 1{ }^{\circ} \mathrm{C}$, and humidity $=50 \pm 5 \%$. The friction on the following solid surface was also measured under similar conditions as the human skin: bioskin plate (S1, artificial skin, urethane elastomer, thickness $=5 \mathrm{~mm}$, Beaulux, Saitama, Japan), acrylic resin ( $\underline{\mathbf{S} 2}$, thickness $=5$ mm, Acrysunday Co., Ltd., Tokyo, Japan), and polytetrafluoroethylene $(\underline{\mathrm{S} 3}$, Naflon PTFE, thickness $=0.1 \mathrm{~mm}$, NICHIAS Corporation, Tokyo, Japan).

\subsection{Skin evaluation}

The moisture content in the stratum corneum and the skin elasticity were measured with a moisture meter (Corneometer CM825TM, Integral Corporation, Tokyo, Japan) and a skin viscoelasticity meter (Cutometer SEM575TM, Integral Corporation, Tokyo, Japan). Moisture content is an arbitrary unit between 0 and 120 . With a skin viscoelasticity measuring instrument, the degree of skin elongation(Uf: maximum height of skin) when absorbed for 2 seconds with a negative pressure of $300 \mathrm{hPa}$ from a suction port, with an inner diameter of $2 \mathrm{~mm}$ and the return of elongation after 0.1 sconds of opening (Ur) was used to obtain a parameter $(\mathrm{Ur} / \mathrm{Uf} \times 100 \%)$ that reflects elasticity. All measurements were performed under conditions of temperature $25 \pm 1{ }^{\circ} \mathrm{C}$ and humidity $50 \pm 5 \%$.

\section{Results}

Figure 2 shows a typical friction profile when the skin is rubbed with the urethane contact probe. The parameter $T /$ $T_{0}$ is the ratio between the round-trip and elapsed times. Normalization of the time by the round-trip time allows
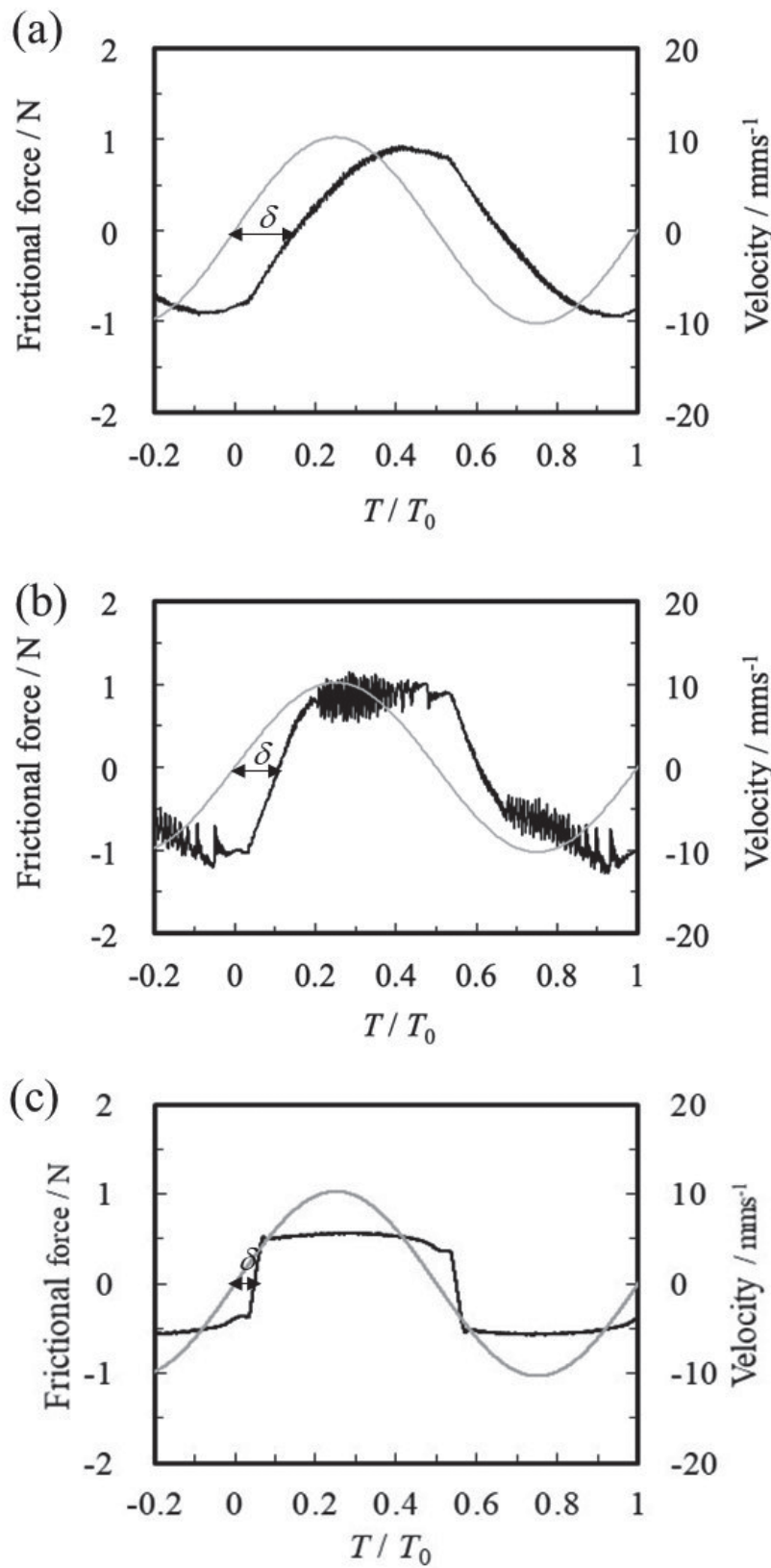

Fig. 2 Temporal profile of the friction force (black line) and velocity (grey line): (a) stable pattern at the fingertip 1 of ID14, (b) stick-slip pattern at the palm $\underline{3}$ of ID14 and (c) bioskin plate $\underline{\mathrm{S} 1}$. 
comparison between data measured at different velocities and sliding width. A stable pattern, wherein the friction profile on the return path was almost identical to that on the forward path, was obtained under all conditions. However, the stick-slip phenomenon, wherein friction force fluctuates periodically, can occur depending on the subject and site. Figure 2 (a) shows a stable profile without the occurrence of the stick-slip phenomenon. The fingertip 1 of the subject ID 14 was rubbed at an angular velocity of $2.1 \mathrm{rad} \mathrm{s}^{-1}$ with a vertical load of $0.98 \mathrm{~N}$. The friction coefficient increased with the increase in sliding velocity and reached 0.97 at $T / T_{0}=0.409$. A delay was observed in the frictional response to the movement of the contact probe. The delay time $\delta$ was normalized by dividing it by $T_{0}$ to yield a value of 0.148 . Time $\delta$ is important in evaluating the frictional properties on the soft material surface, because this parameter reflects the viscoelasticity of the soft material. Figure 2(b) shows a typical profile with a stick-slip pattern. The palm $\underline{3}$ of subject ID 14 was rubbed with a vertical load of $0.98 \mathrm{~N}$ at an angular velocity of $2.1 \mathrm{rad} \mathrm{s}^{-1}$. The friction coefficient reached 0.73 at $T / T_{0}=0.373$ and oscillated with a width of approximately 0.5 . The delay time $\delta$ was 0.112 . Figure 2(c) shows the friction profile of the bioskin plate $(\underline{\mathrm{S} 1})$ as a typical soft material. The profile was the stable pattern, in which the friction coefficient increased with the increase in sliding velocity and reached 0.52 at $T / T_{0}=0.051$ and the delay time $\delta$ was 0.045 .

Next, we obtained the friction coefficient, delay time $\delta$, and occurrence rate of the stick-slip phenomenon to show the characteristic points of human skin. The occurrence rate is the number of times the stick-slip pattern is observed per 60 evaluations. Therefore, measurements were performed thrice for each of the 20 subjects to provide 60 measurements. These parameters indicate that the large delay time and an occasional stick-slip phenomenon are characteristics of skin friction dynamics.

The friction coefficients at points $\underline{1}-\underline{6}$ were $0.360 \pm$ $0.085,0.302 \pm 0.061,0.444 \pm 0.112,0.141 \pm 0.048,0.166 \pm$ 0.053 , and $0.233 \pm 0.076$, respectively (Fig. 3 ). All the readings were smaller than the reading $(1.511 \pm 0.229)$ for acrylic resin $\mathbf{S 2}$ with high surface energy and frictional resistance but were similar to or higher than the results $(0.134 \pm 0.019)$ for polytetrafluoroethylene $\underline{\mathbf{S} 3}$ with low surface energy and frictional resistance. Additionally, the friction coefficients of the finger or palm points $(\underline{1}-\underline{3})$ were larger than those of the forearm points $(\underline{4}-\underline{6})$.

The delay times for points $1-\underline{6}$ were $0.138 \pm 0.048,0.119$ $\pm 0.042,0.122 \pm 0.045,0.202 \pm 0.061,0.188 \pm 0.052$, and $0.158 \pm 0.056$, respectively (Fig. 3 ). These values were extremely large compared with those of the soft materials we have reported so $\mathrm{far}^{11-16)}$. Additionally, the occurrence rates of the stick-slip phenomenon were $65 \%, 93 \%, 85 \%$, $35 \%, 70 \%$, and $80 \%$ for points $1-\underline{6}$, respectively; however, this phenomenon did not occur for materials bioskin plate

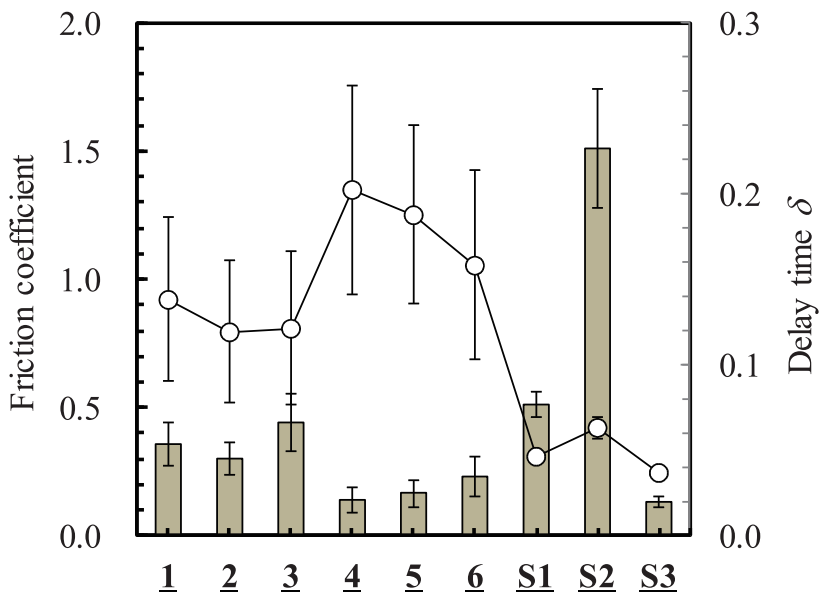

Fig. 3 Friction parameters of skin surfaces $\underline{1}-\underline{6}$ and of materials S1-S3: average of friction coefficient (grey bar) and delay time $\delta$ (white circle).

Table 1 Water content in the skin stratum corneum and skin elasticity.

\begin{tabular}{ccc}
\hline Friction Point & $\begin{array}{c}\text { Water content of stratum } \\
\text { corneum }\end{array}$ & Elasticity \\
\hline$\underline{\mathbf{1}}$ & $47.1 \pm 21.7$ & $19.5 \pm 10.1$ \\
$\underline{\mathbf{2}}$ & $25.5 \pm 11.4$ & $37.0 \pm 12.4$ \\
$\underline{\mathbf{3}}$ & $28.6 \pm 14.1$ & $43.2 \pm 14.0$ \\
$\underline{\mathbf{4}}$ & $32 \pm 5.7$ & $48.9 \pm 13.1$ \\
$\underline{\mathbf{5}}$ & $31.1 \pm 7.2$ & $69.8 \pm 9.4$ \\
$\underline{\mathbf{6}}$ & $32.3 \pm 9.0$ & $75.6 \pm 7.6$ \\
$\underline{\mathbf{S 1}}$ & $6.2 \pm 2.1$ & $74.1 \pm 13.3$ \\
$\underline{\mathbf{S}}$ & $0 \pm 0$ & - \\
$\underline{\mathbf{S 3}}$ & $0 \pm 0$ & - \\
\hline
\end{tabular}

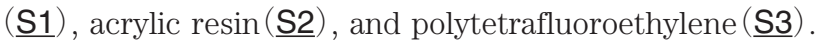
We observed both a stable pattern and a stick-slip pattern when the fingertip was rubbed with the probe, because the friction pattern depends on the surface shape, physical and mechanical properties of human skin.

The effects of water content in the skin stratum corneum and skin elasticity were examined (Table 1). The moisture content at fingertip 1 , $47.1 \pm 21.7$ (A.U.), was higher than those at points $\underline{2}$ to $\underline{6}$ : 26-32 (A.U.). However, all these values were higher than the water content of $\underline{\mathbf{S}}$, which was $6.2 \pm 2.1$ and those of $\underline{\mathbf{S}} \mathbf{2}$ and $\underline{\mathbf{S}} \mathbf{3}$, which were 0 . This concludes that the skin contains greater amount of water than any general soft material, and the water content of the fingertip is particularly significant. The elastic parameter Ur/Uf of points $\underline{1}-\underline{6}$ were $37.0 \pm 12.4 \%, 43.2 \pm$ $14.0 \%, 48.9 \pm 13.1 \%, 69.8 \pm 9.4 \%, 75.6 \pm 7.6 \%$, and $74.1 \%$ $\pm 13.3 \%$, respectively. The definition of the elastic parameter is described as follows: Uf and Ur are the degree of skin elongation and the return of elongation, respectively. 


\section{Y. Nonomura and H. Ouchi}

The elastic parameter of $\underline{\mathrm{S} 1}$ was $74.1 \pm 13.3 \%$; those of $\underline{\mathrm{S} 2}$ and $\underline{\text { S3 }}$ were not measured. The elastic parameter Ur/Uf of $\underline{4} \underline{6}$ was larger than that of $\underline{1}-\underline{3}$ : The reversible return of deformation to mechanical stimuli is greater for the locations $\underline{4}-\underline{6}$.

\section{Discussion}

A significant delay time $\delta$ was observed on the skin surface. The time of the skin surfaces $\underline{1}-\underline{6}$ was several times longer than that of common soft matter surfaces $\underline{\text { S1-S3 }}$. We guess that the large $\delta$ is caused by the characteristic deformation behavior of human skin during the sliding process. We observed that the contact probe significantly deformed the skin surface horizontally during the early stages of sliding because the soft and adhesive skin has a layered structure.

A stick-slip phenomenon occurred on the skin surface. Since the skin exhibits viscoelasticity, the contact probe deforms and sinks into the skin. It is considered that stickslip phenomenon was observed only on the skin surface because the contact probe sticks to the skin and is repeatedly peeled off. Finally, the friction coefficient and delay time $\delta$ differed between the finger/palm, and forearm: The friction coefficient on the finger and palm was $0.3 \sim 0.4$ and larger than that found on the forearm, which was $0.1 \sim 0.2$. Additionally, the delay time averaged $0.11 \sim 0.14$ for the finger/palm and $0.16 \sim 0.20$ for the forearm, and the value for the forearm was larger than that of the finger/palm. This is probably because mechanical properties and deformation behavior depend on the location of the skin surface. In the future, the relationship between skin deformation and friction phenomena will be analyzed in detail using a combinatorial system of friction evaluation system with a high-speed camera.

\section{Conclusions}

Friction force was evaluated on skin surface using a sinusoidal motion friction evaluation system. The friction coefficients were $0.14-0.44$, while delay time $\delta$ was $0.12-0.20$ : the time $\delta$ was significantly larger than that of common soft materials. In addition, we observed a stick-slip phenomenon during the sinusoidal motion friction processes. These dynamic phenomena are due to the softness and viscoelastic properties of human skin.

\section{Acknowledgment}

This study was supported by a Grant-in-Aid for Scientific Research on Innovative Areas (Grants No. 16H01661) and
Scientific Research (B) (Grants No. 18H01402) from the Ministry of Education, Culture, Sports, Science, and Technology, Japan (MEXT).

\section{References}

1) Cosmetic Science and Technology: Theoretical Principles and Applications (Sakamoto, K.; Lochhead, R.Y.; Maibach, H. eds.). Elsevier, Amsterdam, Chapter 4 (2017).

2) Honda, H.; Imayama, S.; Tanemura, M. A fractal-like structure in the skin. Fractals 4, 139-147 (1996).

3) Maeno, T.; Kobayasshi, K.; Yamazaki, N. Relationship between the structure of human finger tissue and the location of tactile receptors. JSME Int. C 41, 94-100 (1998).

4) Caspers, P.J.; Lucassen, G.W.; Carter, E.A.; Bruining, H.A.; Puppel, G.J. In vivo confocal Raman microspectroscopy of the skin: Noninvasive determination of molecular concentration profiles. J. Invest. Dermatol. 166, 434-442 (2001).

5) Comaish, S.; Bottoms, E. The skin and friction: deviations from Amonton's laws, and the effects of hydration and lubrication. Br. J. Daramatol. 84, 37-43 (1971).

6) Adams, M.J.; Briscoe, B.J.; Johnson, S.A. Friction and lubrication of human skin. Tribol. Lett. 26, 239-253 (2007).

7) Nonomura, Y.; Fujii, T.; Arashi, Y.; Miura, T.; Maeno, T.; Tashiro, K.; Kamikawa, Y.; Monchi, R. Tactile impression and friction of water on human skin. Colloids Surf. B 69, 264-167(2009).

8) Nonomura, Y.; Miura, T.; Miyashita, T.; Asao, Y.; Shirado, H.; Makino, Y.; Maeno, T. How to identify water from thickener aqueous solutions by touch. J. R. Soc. Interface 9, 1216 (2012).

9) Aita, Y.; Nonomura, Y. Friction and surface temperature of wet hair containing water, oil, or oil-in-water emulsion. J. Oleo Sci. 65, 493-498(2016).

10) Barnes, H.A.; Hutton, J.T.; Walters, K. An Introduction to Rheology. Elsevier, Amsterdam, Chapter 3 (1989).

11) Aita, Y.; Asanuma, N.; Takahashi, A.; Mayama, H.; Nonomura, Y. Nonlinear friction dynamics on polymer surface under accelerated movement. AIP Adv. 7, 045005 (2017).

12) Aita, Y.; Asanuma, N.; Mayama, H.; Nonomura, Y. Sliding profile and energy at static friction between polymer surfaces. Chem. Lett. 47, 767-469 (2018).

13) Kikegawa, K.; Kuhara, R.; Kwon, J.; Sakamoto, M.; Tsuchiya, R.; Nagatani, N.; Nonomura, Y. Physical origin of a complicated tactile sensation: 'shittori feel'. R. Soc. Open Sci. 6, 190039 (2019). 
14) Tsuchiya, R.; Kuhara, R.; Kikegawa, K.; Nagatani, N.; Nonomura, Y. Tactile and physical properties of cosmetic powders with a shittori feel. Bull. Chem. Soc. Jpn. 93, 399-405 (2020).

15) Shinomiya, K.; Mayama, H.; Nonomura, Y. Anomalous friction between agar gels under accelerated motion. Langmuir 34, 12723-12729 (2018) .

16) Okawara, H.; Shinomiya, K.; Nonomura, Y. Friction dynamics on rough agar gel surfaces. J. Oleo Sci. 68, 873-879 (2019). 